\title{
First operation in SPIDER and the path to complete MITICA
}

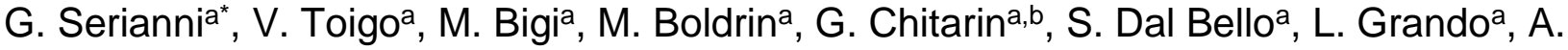
Luchetta $^{a}$, D. Marcuzzia ${ }^{a}$ R. Pasqualotto ${ }^{a}$, N. Pomaro ${ }^{a}$, P. Zaccariaa, L. Zanotto ${ }^{a}$, P. Agostinettia, M. Agostini ${ }^{\mathrm{a}}$, V. Antoni ${ }^{\mathrm{a}}$, D. Aprile ${ }^{\mathrm{a}, \mathrm{c}}, \mathrm{M}$. Barbisan ${ }^{\mathrm{a}}, \mathrm{M}$. Battistella ${ }^{\mathrm{a}}, \mathrm{M}$. Brombin ${ }^{\mathrm{a}}$, A. Canton ${ }^{\mathrm{a}}$, R. Cavazzana ${ }^{a}$, M. Dalla Palma ${ }^{a}$, M. Dan ${ }^{a}$, R. Delogua ${ }^{a}$ A. De Lorenzi ${ }^{a}$, M. De Muria , S. Denizeaua, M. Fadone ${ }^{a}$, F. Fellina, A. Ferro, E. Gaioa, G. Gambetta ${ }^{a}$, F. Gasparini ${ }^{a}$, F. Gnesotto ${ }^{a}$, P. Jain ${ }^{a}$, A. Maistrelloa, G. Manduchia, S. Manfrina, G. Marchioria, N. Marconato ${ }^{a}$, M. Moresco ${ }^{a}$, T. Pattona , M. Paveia , S. Peruzzo ${ }^{a}$, N. Pilan ${ }^{a}$, A. Pimazzonia,c, R. Piovan ${ }^{a}$, C. Poggia, M. Recchia ${ }^{a}$, A. Rigoni ${ }^{a}$, A. Rizzolo a , G. Rostagni ${ }^{a}$, E. Sartoria, , M. Siragusa ${ }^{a}$, P. Sonato ${ }^{a}$, E. Spada ${ }^{a}$, S. Spagnoloa, M. Spolaore ${ }^{a}$, C. Taliercio ${ }^{a}$, P. Tintia , M. Ugolettia, M. Valente ${ }^{a}$, A. Zamengo ${ }^{a}$, B. Zaniola $^{a}$, M. Zaupa ${ }^{a}$, M. Cavenago ${ }^{c}$, D. Boilson ${ }^{d}$, C. Rottid, P. Veltri ${ }^{d}$, J. Chareyre ${ }^{d}$, H. Decamps ${ }^{d}$, M. Dremeld, J. Graceffa ${ }^{d}$, F. Gelid, B. Schunke ${ }^{d}$, L. Svenssond ${ }^{d}$, M. Urbanid , T. Bonicellie, G. Agaricie, A. Garbuglia ${ }^{e}$, A. Masiello, F. Paoluccie , M. Simone, L. Bailly-Maitre ${ }^{e}$, E. Bragulate, G. Gomez $^{\mathrm{e}}$, D. Gutierrez ${ }^{\mathrm{e}}$, C. Labate ${ }^{\mathrm{e}}$, G. Mico ${ }^{\mathrm{e}}$, J.F. Moreno ${ }^{\mathrm{e}}$, V. Pilarde ${ }^{\mathrm{e}}, \mathrm{G}_{\text {. Kouzmenko }}{ }^{\mathrm{e}}, \mathrm{A}$.

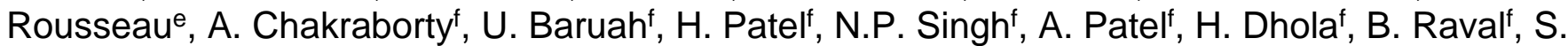

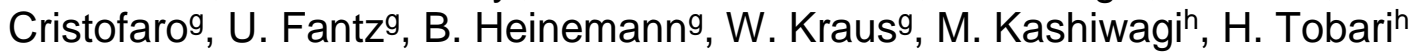

${ }^{a}$ Consorzio RFX (CNR, ENEA, INFN, UNIPD, Acciaierie Venete SpA), Corso Stati Uniti 4 - 35127 Padova, Italy

${ }^{b}$ Università degli Studi di Padova, Dept. of Management and Engineering, Strad. S. Nicola 3, 36100 Vicenza, Italy

'INFN-Laboratori Nazionali di Legnaro (LNL), v.le dell'Università 2, I-35020 Legnaro PD, Italy

${ }^{d}$ ITER Organization, Route de Vinon-sur-Verdon, CS 90 046, F-13067 St. Paul-lez-Durance, France

${ }^{e}$ Fusion for Energy, C/o Josep Pla 2, E-08019 Barcelona, Spain

${ }^{f}$ ITER-India, Institute for Plasma Research, Nr. Indira Bridge, Bhat Village, Gandhinagar, Gujarat 382428, India

${ }^{8} I P P$, Max-Planck-Institut für Plasmaphysik, Boltzmannstraße 2, D-85748, Garching bei München, Germany

${ }^{h}$ National Institutes for Quantum and Radiological Science and Technology, 801-1 Mukoyama, Naka, Ibaraki-ken 311-0193, Japan

(Presented XXXXX; received XXXXX; accepted XXXXX; published online XXXXX)

(Dates appearing here are provided by the Editorial Office)

The requirements of ITER neutral beam injectors ( $1 \mathrm{MeV}, 40 \mathrm{~A}$ negative deuterium ion current for one hour) have never been simultaneously attained; therefore, a dedicated Neutral Beam Test Facility (NBTF) was set up at Consorzio RFX (Padova, Italy). The NBTF includes two experiments: SPIDER, the full-scale prototype of the source of ITER injectors, with a $100 \mathrm{keV}$ accelerator, to investigate and optimise the properties of the ion source; MITICA, full-scale prototype of the entire injector, devoted to the issues related to the accelerator, including voltage holding at low gas pressure. The present paper gives an account of the status of the procurements, of the timeline, of the voltage holding tests and experiments for MITICA. As for SPIDER, the first year of operation is described, regarding the solution of some issues connected with the radiofrequency power, the source operation and the characterisation of the first negative ion beam.

\section{INTRODUCTION}

To reach fusion conditions and to control the plasma configuration in ITER, which represents the next step in controlled thermonuclear fusion research, two heating and current-drive neutral beam injectors (NBIs) will supply a total power of 33MW, by accelerating negative deuterium ions to $1 \mathrm{MeV}^{1}$. The requirements of ITER NBIs, in terms of negative ion current (40A) and beam-on time (1h) have never been simultaneously attained.

*Electronic mail: gianluigi.serianni@igi.cnr.it.
Because of this, in the dedicated Neutral Beam Test Facility (NBTF) at Consorzio RFX (Italy) the performances of the ITER NBI (including the beam optics) will be studied and optimised ${ }^{2,3,4}$. The NBTF includes two experiments: MITICA ${ }^{5}$, full-scale ITER NBI prototype, and SPIDER ${ }^{6}$, full-scale prototype of the ITER NBI source with $100 \mathrm{keV}$ particle energy. SPIDER goal is to investigate and optimise the uniformity of the source plasma (over a $1 \mathrm{~m} \times 2 \mathrm{~m}$ area), the current density of the negative ions and to study the beam optics at low energy ${ }^{7}$. When MITICA exploitation will begin, the experience gained in SPIDER will be immediately applied to the operation of the ion source; SPIDER will continue to operate to address source-specific topics in view of the ITER 
NBI, and MITICA will be employed to solve the issues related to the accelerator, including beam optics in ITER-relevant conditions and high voltage holding in vacuum. This way the NBTF will provide several years of experience with the source and the whole injector before the start of ITER NBI and will accompany the ITER NBI operation so as to solve possible injector issues ${ }^{2}$. So the NBTF schedule is tightly linked with the overall ITER staged approach ${ }^{8}$, which assumes both ITER NBIs to be installed by September 2031 . To fulfil such a plan, SPIDER is required to provide most of the relevant information when MITICA will start beam operation (spring 2023), whereas the experience gained with SPIDER and MITICA will have to confirm the design of the beam source of ITER NBIs, whose construction is planned to start by mid-2027.

The present contribution will briefly outline the activities and the experiments carried out in SPIDER during its first year of operation with volume generation of negative hydrogen ions, including the first investigation of a negative ion beam in SPIDER. Moreover, an update will be given about the status of MITICA and of the whole NBTF as well as a brief outline of the activities planned for the next future, both for SPIDER and MITICA.

\section{SPIDER OPERATIONS}

The SPIDER experiment is the full-size plasma source of MITICA (see section III) and of the NBI for ITER; source operation started in May 20187. The design parameters are summarised in table 1 . The SPIDER ion source design ${ }^{9}$ is essentially the same as for MITICA: the plasma is generated inductively by 8 coils powered in pairs by 4 radiofrequency (RF) generators due to provide up to $200 \mathrm{~kW}$ each. The plasma expands into a chamber, which is closed, opposite to the drivers, by the plasma grid (PG), provided with 1280 apertures, arranged into $4 \times 4$ groups. Each group features 5(horizontally) $\times 16$ (vertically) apertures, through which negative ion beamlets are extracted, thanks to the electrostatic field induced by the voltage difference applied with respect to the next grid (extraction grid, EG). Finally, the voltage applied with respect to the last grid (grounded grid, GG) accelerates particles up to the total energy of about $100 \mathrm{keV}$. In accelerators of negative ions, electrons are co-extracted from the plasma together with the negative ions; a suitable arrangement of permanent magnets, housed in the EG, dumps the electrons onto the EG itself; this field however affects also the trajectories of negative ions, so that suitable countermeasures are needed to compensate for such negative ion deflection. The whole magnetic field configuration of SPIDER shares the basic principles with MITICA; in both cases, the compensation of the negative ion deflection is performed by another system of permanent magnets, which are embedded in the EG itself ${ }^{10}$ for MITICA and in the GG for SPIDER (which also requires a ferromagnetic plate on the downstream side of the GG). In both devices, a magnetic filter field is created just upstream with respect to the PG by currents flowing in the PG and in other busbars, in order to reduce the temperature and the density of electrons in the vicinity of the PG and hence the lifetime and density of negative ions. In negative ion beams, the amount of negative ions in the PG region is greatly enhanced by caesium-catalysed conversion of positive hydrogen ions and atoms impinging on the PG. To this purpose, dedicated evaporators (not yet operating in SPIDER) inject caesium into the ion source ${ }^{11}$.

SPIDER is a key asset in the development of the negative-ion beam source for ITER. For the first time, the vacuum-insulated source concept is fully applied to a beam source for fusion: the multi-aperture multi-electrode electrostatic accelerator and the RF- driven plasma source (at high voltage) are completely contained in the same vacuum as the beam drift region (Fig. 1). Electrical insulation from the vessel is achieved by maintaining a sufficiently low gas pressure, to avoid electrical discharges in the Paschen region. Unlike the other existing prototype sources for fusion applications, SPIDER allows to develop procedures and technical solutions that are specific of this vacuum-insulated source concept. In this sense, during the first year of operation, the experimental activities addressed a pressing issue related to electrical insulation in the presence of RF fields, a condition that is more difficult than in steady state. More than one year since the ignition of the first plasma and after a large effort dedicated, in parallel, to the commissioning of diagnostics and to the optimisation of the RF generators, SPIDER routinely operates the negative-ion beam produced in the plasma volume. Presently, the experimental work focusses on concluding the commissioning and on improving the reliability of all SPIDER plants, in view of caesium operation. The vacuum-insulated source concept indeed does not allow accessing any part of the beam source for maintenance or mechanical improvements, without breaking the same vacuum in which the caesium layer is built up and conditioned for negative ion production.

Table 1: Main design parameters of SPIDER.

\begin{tabular}{ll}
\hline Parameter & Value \\
\hline Beam energy [keV] & 100 \\
Max source filling pressure $[\mathrm{Pa}]$ & 0.3 \\
Max deviation from beam uniformity & $\pm 10 \%$ \\
Extracted ion current density $\left[\mathrm{A} / \mathrm{m}^{2}\right]$ & $>355 \mathrm{H}_{2} ;>285 \mathrm{D}_{2}$ \\
Beam on time & 1 hour \\
Co-extracted electron fraction & $<0.5\left(\mathrm{H}_{2}\right) ;<1\left(\mathrm{D}_{2}\right)$ \\
\hline
\end{tabular}

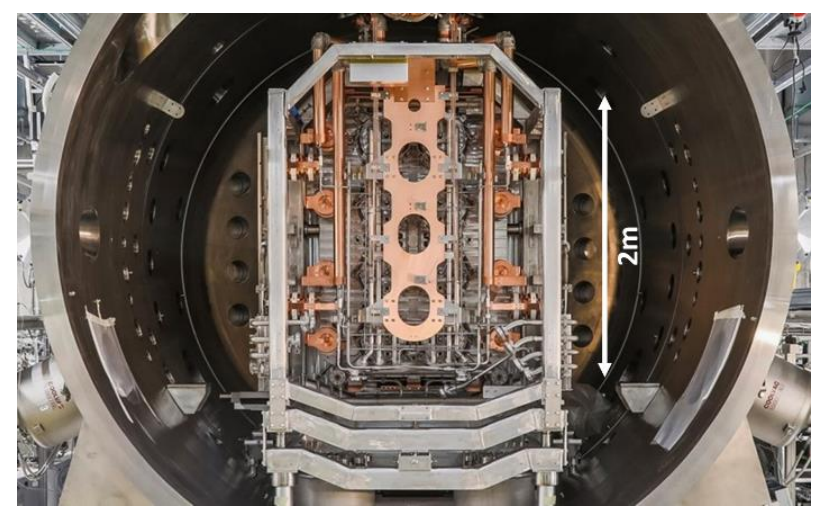

Fig. 1. Rear view of SPIDER beam source in vacuum vessel.

\section{II.a Identification of a solution for the RF-induced discharges}

During SPIDER operations in $2018^{7}$ two interrelated issues emerged, regarding the difficulty in increasing the RF power applied to the RF coils and the occurrence of RF-induced discharges on the rear side of the beam source ${ }^{12}$. These issues impeded simultaneous operation of all RF drivers as well as the operation above a total RF power of $\sim 100 \mathrm{~kW}$. A strategy was developed to identify and address the cause of the problem. Based on the experience of the ELISE test facility ${ }^{13}$, the cause might be the triple points between different materials, where RF electric fields develop, which can induce breakdowns at large RF power. On the other hand, the very RF voltage at components of the resonant circuit (the matching network that is located on the rear side of the source, namely in vacuum) can induce breakdowns at unfavourable gas pressures. It was decided to verify whether the latter was the primary cause, by designing an experiment with 
reduced vessel pressure within a wide range of source pressures. This was attained by installing a plate inside the accelerator so as to close all the apertures of the EG, resulting in a drastic reduction of the gas conductance from the source to the vessel. This experiment showed that, in the present operating conditions (up to $\sim 100 \mathrm{~kW}$ per generator), the dominant effect is related to the gas pressure: it was recognised that above a vessel pressure of $40 \mathrm{mPa}$ the probability of discharges quickly increases; without the plate, such a pressure would correspond to only $0.1 \mathrm{~Pa}$ inside the ion source. In parallel to the definition of a long-term major modification of SPIDER pumping system, (to this purpose also non-evaporable getters are being considered ${ }^{14}$ ), it was decided to carry on with the experimental programme, upon reducing the gas conductance between the source and the vessel by installing a mask on the downstream side of the plasma grid. The mask features 80 apertures (out of 1280); Fig. 2 shows the aperture arrangement in each grid segment (corresponding to $1 / 4$ of a grid). The computation method adopted to model the gas pressure profile, based on the view factor technique, is described in ${ }^{15}$, the mechanical design and installation can be found in ${ }^{16}$, and the electrical considerations and tests are exposed in ${ }^{17}$.
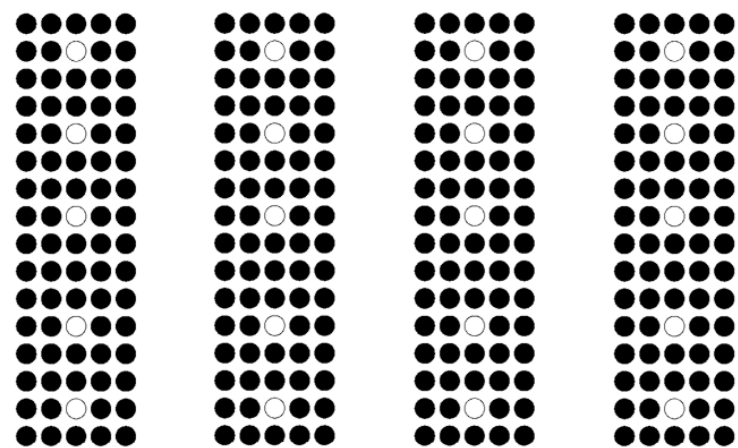

Fig. 2 Arrangement of apertures in the segments of the SPIDER accelerator grids. White circles indicate operating beamlets.

\section{II.b Source and beam characterisation}

After a shutdown started at the end of November 2018, including the installation of the plasma grid mask, SPIDER operations resumed at mid-April 2019 and were devoted to the following activities: characterisation of the RF circuits; characterisation of the ion source; test of the extraction and accelerator power supplies; first characterisation of the SPIDER beam. As of 2019 most of SPIDER diagnostic systems ${ }^{18}$ are operating: the list of source diagnostics ${ }^{19}$ include source emission spectroscopy, surface and calorimetry thermocouples, $\mathrm{H}_{\text {alpha }}$ detectors, visible cameras and electrical measurements at the various power supplies. The electrostatic sensors embedded in the plasma grid and in the bias plate ${ }^{20}$ are under commissioning; the diagnostic systems more tightly related with caesium operation, like laser absorption spectroscopy ${ }^{21}$ and cavity ring-down spectroscopy, will soon be installed and commissioned. The operating diagnostic systems allowed the measurement of the plasma and beam parameters in different experimental conditions, which can be explored even within single pulses thanks to the very flexible SPIDER control system ${ }^{22}$; an overview of the results of the experimental campaigns is given in the following.

A first characterisation of the RF circuits was carried out in parallel to the implementation of a feedforward control of the internal capacitors of the RF generators and to the development of a model reproducing the behaviour of the RF system. The experiments focussed on the parallel capacitance of the matching network, after the temporary installation of different capacitors on the 4 circuits $(5 \mathrm{nF}, 6.5 \mathrm{nF}, 10 \mathrm{nF}, 15 \mathrm{nF})$. The experiments confirmed the presence of hysteresis resulting in a "forbidden" region of frequencies (unfortunately the optimal frequency for transfer of RF to the coils lies in this region) and provided a benchmark for the numerical simulations of the RF circuits. Thus it was possible to thoroughly assess the features of the generators, although in single-generator operation only, so as to attain an RF power per generator up to $125-145 \mathrm{~kW}$ (depending on the generator). Based on this campaign and on the numerical codes, it was subsequently possible to simultaneously and reliably operate the $4 \mathrm{RF}$ generators up to $100 \mathrm{~kW}$ per generator, after restoring the original $10 \mathrm{nF}$ parallel capacitance of all matching networks. Increasing the RF power above that value will be the subject of further investigations (sect. II.c).

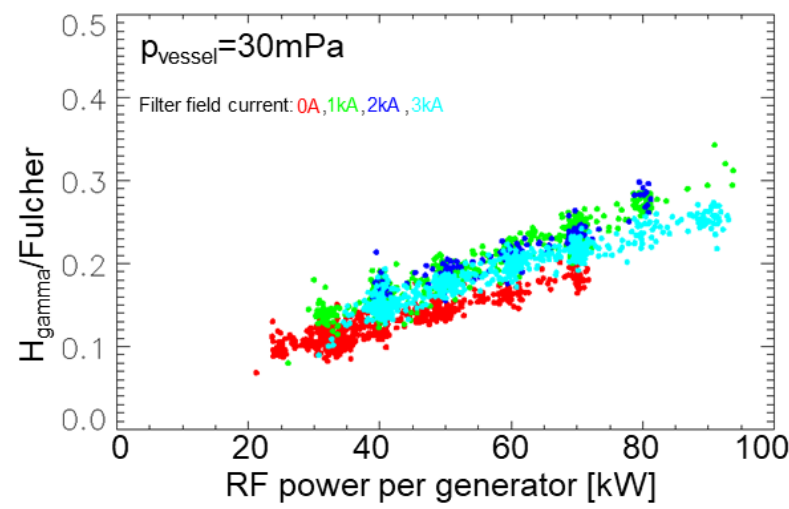

Fig. 3. Ratio of $\mathrm{H}_{\text {gamma }}$ and Fulcher brightness versus $\mathrm{RF}$ power for different values of the magnetic filter field current.

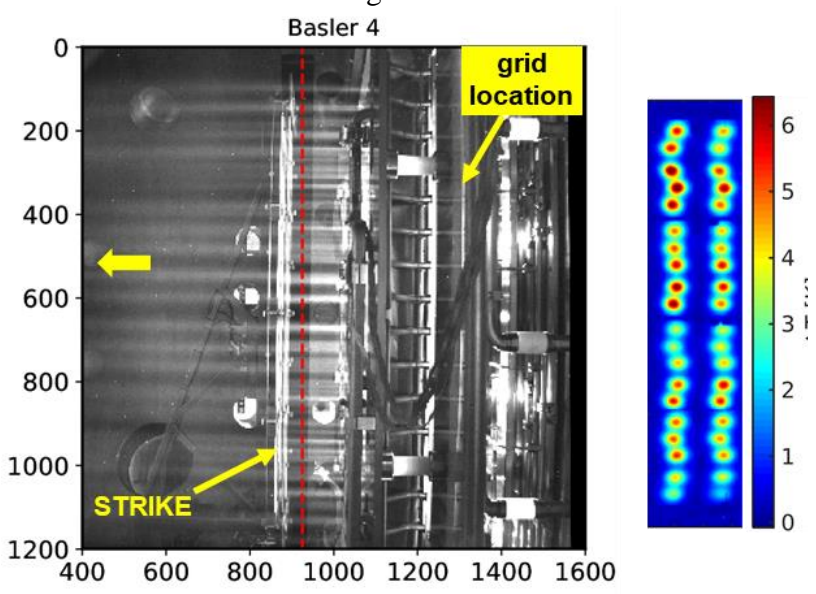

Fig. 4. Image of the beam provided by the visible camera (left); the positions of STRIKE and of the accelerator grids are indicated; the arrow indicates the beam direction. Footprint of the beamlets onto the STRIKE tiles (right).

As for the characterisation of the ion source, signals from $\mathrm{H}_{\text {alpha }}$ detectors ${ }^{23}$ and emission spectra ${ }^{24}$ indicate that, both in the drivers and close to the plasma grid, plasma emission increases with RF power. The plasma completely fills the drivers and the expansion chamber; plasma emission seems to be slightly larger at the bottom of the ion source, but this statement deserves further investigation. The ratio between $\mathrm{H}_{\text {gamma }}$ and Fulcher band seems to increase with the RF power (Fig. 3), suggesting a corresponding increase of the dissociation degree of hydrogen. The rotational 
temperature of the hydrogen molecules increases with RF power and gas pressure. No major influence of electron and beam extraction on the plasma surrounding the PG is suggested by spectroscopy (but it shall be remembered that no caesium was used in SPIDER to enhance the negative ion amount yet). The magnetic filter field was found to affect the plasma even far away from the plasma grid; the light emitted by the plasma reaches a maximum for a certain value of the filter field current (between 600A and $1500 \mathrm{~A}$, depending on pressure and RF power) and decreases for larger values (at low RF power the plasma can be even switched off for large filter field). This evidence was ascribed to the specific topology of the magnetic filter field, which features magnetic field lines intersecting the walls of the RF drivers, thus distorting the electron trajectories, as confirmed by the increase of the heat deposited onto these surfaces as the filter field increases. The magnetic configuration will be improved in the future (sect. II.c).

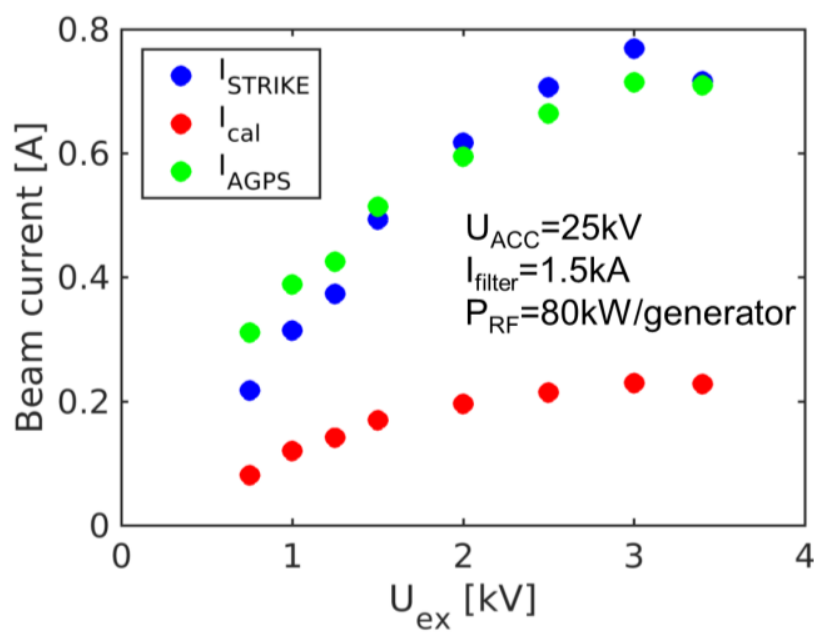

Fig. 5. Dependence of the electrical beam currents (measured at the power supply and on the STRIKE tiles) and of the equivalent calorimetrical current on the extraction voltage.

The first negative ion beam was extracted from the SPIDER ion source and accelerated at the end of May 2019. The signature of the negative hydrogen beam can be clearly seen by the light emitted when the beam interacts with the background gas (Fig. 4, left). Afterwards a first characterisation was performed of the beam features and of the amount of co-extracted electrons. The bias voltages applied to plasma grid and to bias plate were found to affect the amount of co-extracted electrons. Combination of the two voltages, such that the bias plate is slightly more positive than the plasma grid, seems more effective (due to the presently limited voltage range of the bias power supplies, these experiments were performed only at low RF power; this voltage limitation will soon be removed, as described in sect. II.c). As in other devices ${ }^{25}$, coextracted electrons decrease with increasing gas pressure. The magnetic filter field is found to selectively reduce the amount of co-extracted electrons with respect to the negative ions.

The SPIDER beam features can be studied by means of several diagnostic systems: apart from the electrical measurements provided by the power supplies, dedicated diagnostics include visible cameras (constituting the first set of cameras of the beam tomographic system ${ }^{26}$ ), beam emission spectroscopy and the Short-Time Retractable Instrumented KalorimEter (STRIKE) ${ }^{27}$; an emittance scanner is also under preparation ${ }^{28,29}$. The electrical measurement of the beam current at the accelerator power supply and the electrical current collected by STRIKE (located at $0.7 \mathrm{~m}$ from the grounded grid) were found to be comparable and to increase with the extraction voltage (Fig. 5). The current equivalent to the beam energy flux measured calorimetrically at STRIKE confirms the same trend. As expected, the calorimetrical estimate of the negative ion current is lower than the electrical measurement, because the latter includes some of the secondary electrons belonging to the plasma generated by the interaction of the negative ion beam with the background gas. Interpreting the specific value of the ratio between the two estimates (Fig. 5 shows a factor of 2.5 during this first beam characterisation) requires numerical simulations of the beam plasma, which will be addressed in the future.

The installation of the plasma grid mask in SPIDER leaves only isolated beamlets, for a total number of 80 beamlets out of 1280. This configuration of the accelerator gives a unique, albeit temporary, opportunity for the detailed investigation of the beamlet properties. The beam divergence can be measured in SPIDER by several diagnostic systems. Many visible cameras are installed on the vacuum vessel in different positions and with different field of view (Fig. 4, left); the Doppler-shifted light emitted by the beam particles is measured by beam emission spectroscopy along several lines-of-sight; the STRIKE calorimeter records the thermal footprints of the single beamlets on the CFC tiles (Fig. 4, right). Similar values of the beamlet divergence, down to $20-30 \mathrm{mrad}^{27}$, were estimated by the various beam diagnostics and the same dependency on the extraction voltage was found.

\section{II.c Future experimental plan (end of 2019-2020)}

In the fourth quarter of 2019 and in 2020, until the 2020 shutdown, SPIDER experiments will aim at caesium operation and its preparation. A milestone towards caesium operation is the commissioning and testing of all and each of the three caesium ovens. Caesium evaporation from each caesium oven is being demonstrated and characterised ${ }^{30}$ in the dedicated facility CATS ${ }^{11}$. In the next phase, a functional test of the ovens installed in SPIDER is planned, to demonstrate the reliability of the mechanical and electrical design, despite high voltage and RF environment. Like MITICA and the NBI for ITER, wiring and feedthroughs for the caesium ovens lie beside high voltage and RF connections in the transmission line connecting the source to the high voltage deck (HVD).

An RF optimisation campaign will exploit the installation of a variable capacitor and will aim at searching for the maximum $R F$ power attainable with fine control of the capacitance of the matching network (single-generator operation) and at comparing and improve the numerical models of the RF circuitry. Subsequently, strategies to increase the maximum reliable RF power with 4 generators simultaneously operating will be tested.

As already discussed in sect. II.b, presently, the specific configuration of the magnetic filter field of SPIDER, which exhibits an X-point inside the RF drivers, globally affects the plasma; at the beginning of 2020, the filter field will be improved. Moreover the range of the bias voltages (for plasma grid and bias plate) will be increased to make it suitable also for operation with volume generation of negative ions, which is characterised by a higher plasma potential. Then the source parameters will be investigated in detail by introducing a movable system of electrostatic sensors (single and double Langmuir probes; Mach 
probes; retarding field energy analysers) and by adding impurities to the process gas. A campaign will be specifically devoted to the study of the deuterium plasma. A second investigation will be carried out of the electron and beam features as functions of the control parameters (RF power, gas pressure, magnetic filter field, bias voltages, process gas).

In addition to these investigations, the following activities are planned specifically to prepare the caesium operation: definition of a regeneration procedure for cryogenic pumps minimising caesium layer pollution; definition of a reliable high voltage conditioning procedure for the accelerator; commissioning of the cooling of the beam dump; commissioning of laser absorption and of cavity ring-down spectroscopy; demonstration that a cumulative beam and source pulse length per day suitable for a good source caesiation can be attained; demonstration of plasma grid temperature control, to maintain it at about $150^{\circ} \mathrm{C}$ during caesium operation. In parallel the caesium-related diagnostic systems are being tested in the CATS facility ${ }^{31}$ and simulations of the expected behaviour of the caesium evaporators ${ }^{32}$ and of caesium dynamics in vacuum are being carried out ${ }^{33}$.

The caesium campaign will be divided into two parts. First the experimental programme will aim at verifying the improvement of the SPIDER performances as caesium is injected. Particularly, investigations will address the effect of the caesium injection rate and conditioning procedure, since these parameters are probably specific for a source design and size; the recovery after overnight and weekend breaks will also be assessed. The recovery after cryogenic pump regeneration will necessarily be studied, because, similarly to the ITER NBIs, SPIDER is the first negative-ion source not including an absolute valve to isolate a well-conditioned ceasiated source from the vacuum vessel, in which cryogenic pumps are regenerated. Finally, after a sufficient increase of negative ion current and a sufficiently reduced coextracted electron current will be attained, the experimentation will be devoted to the study of the influence on the beam features of extraction voltage, RF power, gas pressure, process gas (hydrogen and deuterium), bias voltages (plasma grid and bias plate), strength of the magnetic filter field.

\section{STATUS OF MITICA}

MITICA is the full-scale injector prototype for ITER NBIs and is expected to demonstrate the operation of the whole ITER injector ${ }^{34}$. It represents a large leap forward in terms of beam power and particle energy with respect to the existing NBIs. The main MITICA parameters are given in Table 2: except for the duration of the hydrogen pulses, they correspond to the parameters of the ITER NBIs, as indicated.

Table 2: Main design parameters for MITICA; the difference with the ITER NBI is indicated.

\begin{tabular}{ll}
\hline Parameter & Value \\
\hline Beam energy [keV] & $1000\left(\mathrm{D}_{2}\right) ; 870\left(\mathrm{H}_{2}\right)$ \\
Max source filling pressure [Pa] & 0.3 \\
Beamlet divergence [mrad] & $\leq 7$ \\
Accelerated current [A] & $46 \mathrm{H}_{2} ; 40 \mathrm{D}_{2}$ \\
Beam on time & 1 hour $\left(\mathrm{H}_{2}\right.$ in ITER: 1000s $)$ \\
Co-extracted electron fraction & $<0.5$ in $\mathrm{H}_{2} ;<1$ in $\mathrm{D}_{2}$ \\
\hline
\end{tabular}

MITICA shall provide the particles with $1 \mathrm{MeV}$ total energy; its physics design is described in ${ }^{35}$ and the status as of 2018 was reported in ${ }^{36}$. The components of the MITICA injector are shown in Fig. 6. The MITICA ion source is essentially the same as for SPIDER (see sect. II). The accelerator is composed of 5 stages, $200 \mathrm{kV}$ each, up to the total particle energy. The magnetic field configuration of MITICA shares the basic principles with SPIDER, although the compensation of the negative ion deflection is performed by another system of permanent magnets embedded in the EG itself ${ }^{10}$.

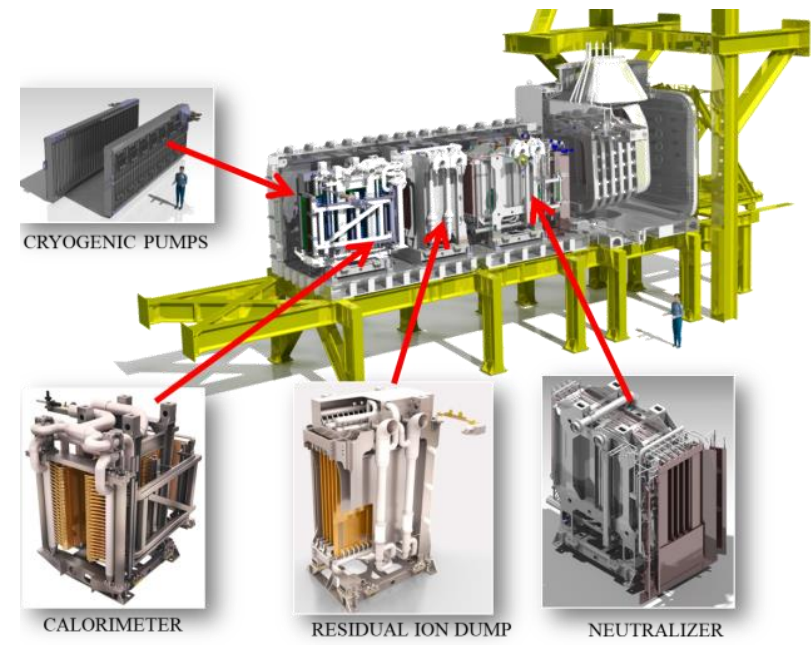

Fig. 6. Structure of the MITICA injector. The beam line components, neutraliser and residual ion dump are highlighted; the beam source (see Fig. 8) can be seen on the right-hand side. The cryogenic pumps are also sketched.
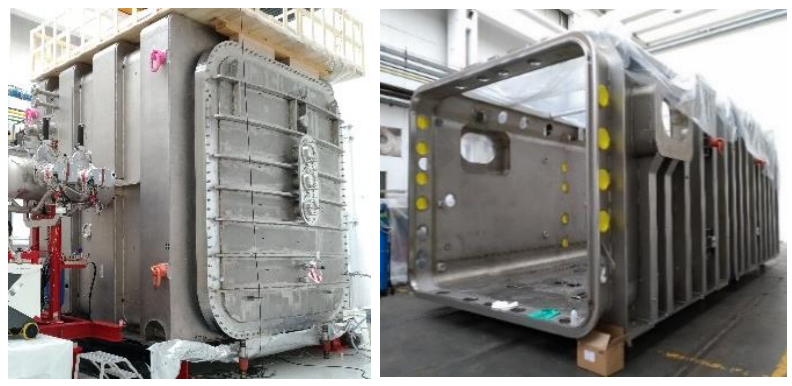

Fig. 7. MITICA beam source vessel under leak test (left) and MITICA beam line vessel under construction (right).

Herein a short account will be given of the main procurement advancements. The MITICA vessel is made of two components, housing the beam source and the beam line components, respectively (Fig. 7). The beam source vessel (Fig. 7, left) was delivered to the NBTF in spring 2019. It was later installed in its final position and provided with a temporary pumping system; the leak test was successfully performed. As for the beam source, which includes the ion source and the accelerator (Fig. 8), the supply contract was signed with the consortium Alsyom-Seiv (France) in October 2018. The first prototypes of the most critical components of the accelerator (Fig. 9, left) are under construction; an instance of a grid support prototype is shown in Fig. 9, right. The beam source should be delivered to the NBTF by mid-2022 .

MITICA beam line includes (Fig. 6): the neutraliser, where negative ions are stripped of their additional electrons by passing through a gas cell; the residual ion dump, to dispose of the negative ions that were not neutralised or which underwent double 
stripping, resulting in positively charged ions; the calorimeter, which receives the neutral beam power for the entire duration of the MITICA experimentation. Moreover two large cryogenic pumps are located on either side of the beam line components to provide the required vacuum conditions in the vessel $\left(p_{\text {vessel }}<20 \mathrm{mPa}\right)$. The supply of the beam line components involves a two-stage contract: stage 1, devoted to the identification of the supplier companies capable of performing the task, is completed; during this stage, prototypes of some of the most critical components were realised. These suppliers will compete for stage 2 , which is under assignment; delivery of the components is planned for the third quarter of 2023.

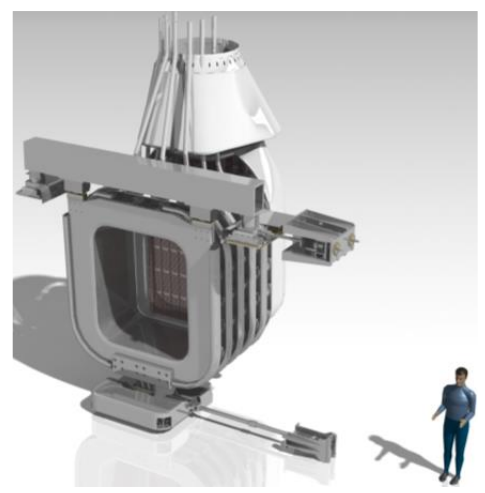

Fig. 8. CAD view of the MITICA beam source.

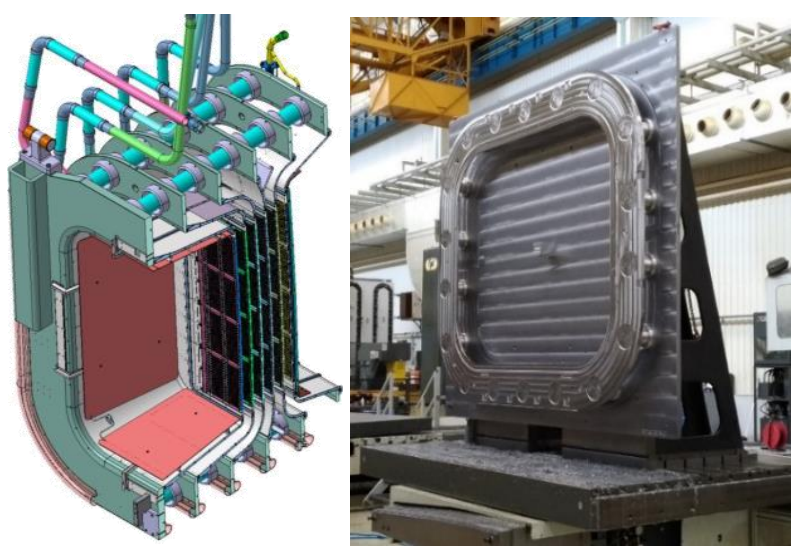

Fig. 9. Cut view of the MITICA accelerator (left). Prototype of the supporting flange of the $4^{\text {th }}$ grid of the MITICA accelerator (right).

The 1MV power supplies (procured in-kind by the Japanese Domestic Agency) are already installed ${ }^{37,38}$; insulation tests and commissioning have started in 2018, and will be concluded with the insulating test of the whole transmission line, including high voltage bushing and vacuum vessel, scheduled for November 2019. The HVD, housing all power supplies at the source potential (including the RF generators), was provided by F4E. In 2019 the combined tests were performed involving the HVD, the $1 \mathrm{MV}$ power supplies and the high voltage transformer (required by the devices housed in the HVD): the integrated insulation tests were successfully performed in July 2019, and consisted of the following steps: $-1.2 \mathrm{MV}$ for 1 hour: $-1.06 \mathrm{MV}$ for $5 \mathrm{~h}$; 5 pulses from $-1.06 \mathrm{MV}$ to $-1.265 \mathrm{MV}$. In 2020 the following activities will be performed: power integrated tests of the $1 \mathrm{MV}$ power supplies (AGPS) at full power on dummy load; completion of commissioning and site acceptance tests of the ion source and extraction power supplies (ISEPS); AGPS-ISEPS power integrated tests; installation and site acceptance tests of the MITICA beam line vessel. In parallel, commissioning and site acceptance tests of the gas and vacuum system will be carried out along with the integration of the plant with the control and protection systems. Starting from 2020, the MITICA power supplies and the vessel, together with an electrostatic mock-up of the source, will be employed to gain experience on high voltage holding in vacuum and at low gas pressure, which is one of the main issues to be addressed in MITICA. Later on, after installing the MITICA beam source inside the vessel, the plasma will be started and characterised in different operational conditions by applying the expertise gained in SPIDER. After delivery and installation of beam line components, beam operation will start.

\section{ACCOMPANYING FACILITIES}

The experimental plan in SPIDER and MITICA is supplemented by an accompanying programme, including some small test facilities at the NBTF. The High Voltage RadioFrequency Test Facility (HVRFTF) ${ }^{39}$ is devoted to the characterisation of the dielectric strength in vacuum of the RF drivers, to address potential voltage holding issues of beam source components under RF electric fields at low gas pressure. In the CAesium Test Stand (CATS) ${ }^{11}$ the characterisation of the caesium ovens before installation into SPIDER and MITICA is performed, in terms of diagnostics, oven maintenance and caesium management. The High Voltage Padova Test Facility (HVPTF) ${ }^{40}$ aims at performing optimised experiments of voltage holding up to $1 \mathrm{MV}$ in vacuum and with gas, with tight control of voltage, current, pressure and vacuum quality. The Negative Ion Optimisation facility, phase 1 (NIO1) $)^{41}$, is a versatile multiaperture hydrogen source, with compact and modular design, capable of continuous operation to reproduce the physics conditions of much larger ion sources, so as to readily verify the effects of individual source components/parameters and to compare results with simulations.

\section{SUMMARY}

In this paper a summary of the activities performed at the ITER neutral beam test facility is given. The components of the MITICA device are being procured or have already been delivered; the $1 \mathrm{MV}$ power supplies are being employed for the voltage holding tests that will soon involve a mock-up of the beam source. The SPIDER facility is operating with volume generation of negative ions since more than one year. Several issues were addressed and solved, which allowed the acceleration of negative ions beams. Caesium will soon be injected in SPIDER to improve the beam performances.

\section{ACKNOWLEDGMENTS}

The work leading to this publication has been funded partially by Fusion for Energy (F4E). This publication reflects the views only of the authors, and F4E cannot be held responsible for any use which may be made of the information contained therein. The views and opinions expressed herein do not necessarily reflect those of the ITER organization.

\section{REFERENCES}

1 T. Inoue et al., Fusion Eng. Des. 56-57, 517 (2001). 
2 R.H.S. Hemsworth et al., Rev. Sci. Instrum. 79, 02 C109 (2008).

3 V. Toigo et al., New J. Phys. 19, 085004 (2017).

4 L. Grisham, Fusion Eng. Des. 87, 1805 (2012).

5 V. Toigo V. et al., Nucl. Fusion 55, 083025 (2015).

6 D. Marcuzzi et al., Fusion Eng. Des. 85, 1792 (2010).

7 G. Serianni et al., Fusion Eng. Des. 146, 2539 (2019).

8 B. Bigot, Nucl. Fusion 59, 112001 (2019).

9 P. Agostinetti et al., Nucl. Fusion 51, 063004 (2011).

${ }^{10}$ G. Chitarin et al., Rev. Sci. Instrum. 85, $02 B 317$ (2014).

11 A. Rizzolo et al., Fusion Eng. Des. 146, 676 (2019).

${ }^{12}$ A. Zamengo, M. Agostini, RF Breakdowns in the SPIDER experiment during its first operational phase, presented at MeVArc 2019.

${ }^{13}$ B. Heinemann et al., Fusion Eng. Des. 136, 569 (2018).

${ }^{14} \mathrm{M}$. Siragusa et al., Numerical simulation of experimental tests performed on ZAO ${ }^{\circledR}$ Non- Evaporable-Getter pump designed for NBI applications, presented at ICIS2019, submitted to Rev. Sci. Instrum.

15 E. Sartori et al., Fusion Eng Des. 151, 111398 (2020).

${ }^{16} \mathrm{M}$. Pavei et al., SPIDER Plasma Grid masking for reducing gas conductance and pressure in the Vacuum Vessel, presented at ISFNT 2019; submitted to Fusion Eng. Des.

17 A. Maistrello et al., Voltage Hold Off Test of the Insulating Supports for the Plasma Grid Mask of SPIDER, presented at ISFNT 2019; submitted to Fusion Eng. Des.

${ }^{18}$ R. Pasqualotto et al., JINST 12, C10009 (2017).

${ }^{19}$ R. Pasqualotto et al., AIP Conf. Proc. 1869, 030020 (2017).

${ }^{20}$ M. Brombin et al., Rev. Sci. Instrum. 85, 02 A715 (2014).

${ }^{21}$ M. Barbisan et al., Fusion Eng. Des. 146, 2707 (2019).

22 A. Luchetta et al., Fusion Eng. Des. 146, 500 (2019).

${ }^{23}$ R. Pasqualotto et al., Fusion Eng. Des. 146, 709 (2019).

${ }^{24}$ B. Zaniol et al., Rev. Sci. Instrum. 91, 013103 (2020).

${ }^{25}$ P. Franzen et al., Plasma Phys. Control. Fusion 56, 025007 (2014).

${ }^{26}$ R. Pasqualotto et al., Fusion Eng. Des. 88, 1253 (2013).

${ }^{27}$ A. Pimazzoni et al., Assessment of the SPIDER beam features by diagnostic calorimetry and thermography, presented at ICIS 2019, submitted to Rev. Sci. Instrum.

${ }^{28}$ E. Sartori et al., Plasma Fusion Res. 13, 3405092 (2018).

${ }^{29}$ C. Poggi et al., Design and development of an Allison type emittance scanner for the SPIDER ion source, presented at ICIS 2019, submitted to Rev. Sci. Instrum.

${ }^{30}$ S. Cristofaro et al., Rev. Sci. Instrum. 90, 113504 (2019).

${ }^{31}$ M. Barbisan et al., JINST 14, C12011 (2019).

${ }^{32}$ E. Sartori, Simulation-based quantification of alkali-metal evaporation rate and systematic errors from current-voltage characteristics of Langmuir-Taylor detectors, accepted for publication in IEEE Trans. Instrum. Meas.

${ }^{33} \mathrm{M}$. Fadone et al., Interpreting the dynamic equilibrium during evaporation in a Caesium environment, presented at ICIS 2019, submitted to Rev. Sci. Instrum.

${ }^{34}$ R.H.S. Hemsworth et al., New J. Phys. 19, 025005 (2017).

${ }^{35}$ P. Agostinetti et al., Nucl. Fusion 56, 016015 (2016).

36 V. Toigo et al., Nucl. Fusion 59, 086058 (2019).

${ }^{37} \mathrm{H}$. Tobari et al 2018 Completion of dc 1 MV power supply system for ITER neutral beam test facility, Preprint: 2018 IAEA Fusion Energy Conf. (Gandhinagar, India, 22-27 October 2018) FIP/1-10.

${ }^{38}$ L. Zanotto et al., Fusion Eng. Des. 146, 2238 (2019).

39 A. Maistrello et al., Fusion Eng. Des. 131, 96 (2018).

${ }^{40}$ N. Pilan et. al. Fusion Eng. Des. 88, 1038 (2013).

${ }^{41}$ M. Cavenago et al., Rev. Sci. Instrum. 91, (2020) 013316. 